\title{
Guiding Hand X Rays with Glass Polycapillary Fiber
}

\author{
Q. F. Xiao, I. Yu. Ponomarev, A. I. Kolomitsev, and D. M. Gibson \\ X-Ray Optical Systems, Inc., 1400 Washington Ave., \\ Albany, NY 12222 \\ F. A. Dilmanian, and E. Nachaliel \\ Medical Department, Brookhaven National Laboratory, \\ Upton, NY 11973
}

\begin{abstract}
$\mathrm{X}$ rays can be guided through a polycapillary fiber by multiple total reflections from the smooth channel walls of the fiber. Using monochromatic Synchrotron Radiation at energies of 22 and $44 \mathrm{keV}$, we measured the efficiency of transmission of $x$ rays through polycapillary fibers with channel diameters of about $13 \mu \mathrm{m}$. Efficiencies of $57.3 \%$ and $54.5 \%$ for $22 \mathrm{keV}$ and $44 \mathrm{keV} \times$ rays, respectively, were obtained with a $120-\mathrm{mm}$-long straight polycapillary fiber aligned with the incident beam. These values are close to the open fraction of the fiber, which is about $60 \%$. In addition, transmission efficiency was measured as a function of the tilt angle between the incident beam and the axis of the fiber. We also measured the transmission efficiency as a function of the deflection angle for a 114-mm-long curved polycapillary fiber. The measurements are compared with a ray-tracing simulation.
\end{abstract}

\section{DISCLAIMER}

\begin{abstract}
This report was prepared as an account of work sponsored by an agency of the United States Government. Neither the United States Government nor any agency thereof, nor any of their employees, makes any warranty, express or implied, or assumes any legal liability or responsibility for the accuracy, completeness, or usefulness of any information, apparatus, product, or process disclosed, or represents that its use would not infringe privately owned rights. Reference herein to any specific commercial product, process, or service by trade name, trademark, manufacturer, or otherwise does not necessarily constitute or imply its endorsement, recommendation, or favoring by the United States Government or any agency thereof. The views and opinions of authors expressed herein do not necessarily state or reflect those of the United States Government or any agency thereof.
\end{abstract}


The concept of $\mathrm{x}$-ray steering based on multiple total reflections within a hollow glass capillary has been known for many years [1-5]. $\mathrm{X}$ rays have been focussed on a micrometer scale using a tapered glass capillary [6,7]. However, the use of arrays of curved glass capillaries to collect and steer $\mathbf{x}$-rays was introduced only recently by Kumakhov and his collaborators [8-10]. Subsequently, various novel $x$-ray optical devices, collectively known as Kumakhov lenses, were conceived and demonstrated $[10,11]$. Kumakhov lenses can control $\mathbf{x}$ rays over a broad range of angles and energies and have many potential functions including beam collection and focusing, beam collimation and energy filtering for $\mathrm{x}$-ray imaging systems, projection lithography, and various research applications. The underlying geometric consideration for a Kumakhov lens is that the channel diameters are much less than the radius of curvature of the fibers in the lens, which ensures that, at each reflection, propagating $x$ rays are incident on the channel walls at angles less than the critical angle for total reflection. For a parallel $\mathrm{x}$-ray beam aligned with the entrance of a capillary, this condition can be expressed by [9],

$$
R>\frac{2 d}{\theta_{c}^{2}},
$$

where $R$ is the radius of curvature of the fibers, $d$ is the channel diameter, and $\theta_{c}$ is the critical angle for total reflection. For silica glass, $\theta_{c}$ is about 30/E mrad, where $E$ is the $x$-ray energy in $\mathrm{keV}$. Thus the channel size must be decreased to reduce the physical size of the Kumakhov lenses by decreasing $\mathbf{R}$, or to transmit higher energy photons which have a smaller $\theta_{c}$.

The success of the Kumakhov lens resulted in extensive experimental and theoretical studies of the transmission of $x$ rays in capillaries. Quantitative studies have been made of the transmission of $\mathbf{x}$ rays through polycapillary fibers under different geometries using soft $\mathbf{x}$ rays $(\sim 1 \mathrm{keV})[12]$ and $x$ rays of $8-20 \mathrm{keV}[13,14]$. This paper reports our measurements of the transmission efficiency of hard $x$ rays through straight and curved polycapillary fibers using monochromatic synchrotron radiation at energies of 22 and $44 \mathrm{keV}$. The experimental data are compared with the results of a ray-tracing simulation.

The simulation traces the trajectories of a large number of $x$ rays, so that the distribution of initial trajectories represents the conditions of the experiment. Each incident 
angle of reflection during the passage of an $x$ ray through a capillary is calculated, so that the probability that the $\mathrm{x}$ ray is transmitted is the product of all the reflection probabilities. These probabilities are calculated according to the Fresnel equation for the reflection coefficient, using the complex index of refraction for glass. A detailed description of the simulation program can be found in reference [13].

The measurements reported in this paper were performed at beamline X17B at the National Synchrotron Light Source of Brookhaven National Laboratory. The $x$-ray beam was monochromatized by a double-crystal monochromator ( $\mathrm{Si}(220)$ ) and had a fundamental energy of $22 \mathrm{keV}$, together with a second harmonic at $44 \mathrm{keV}$. A NaI scintillation detector was used to detect the $x$ rays.

The polycapillary fibers used were made of borosilicate glass. The cross-section of the fiber is hexagonal, with $0.44 \mathrm{~mm}$ between opposite corners. Each fiber contains 547 hexagonal channels, each with a diameter of about $13 \mu \mathrm{m}$. The open area of the fiber is about $60 \%$, including small triangular channels. Figure 1 is an optical micrograph of the cross-section of the polycapillary fiber.

For measurements on straight polycapillary fibers, the fibers were placed against the right-angle corner of an _ـ-shaped, optically flat aluminum plate. To measure the 114-mmlong bent fiber, the 19-mm-long entrance part of the fiber was pressed against the corner of the plate with a steel block and the remainder of the fiber was bent by pushing the exit end of the fiber horizontally with a micrometer. For small deflection angles, this simple procedure creates a cubic bending with a curvature which is greatest at the beginning of the bend and which decreases linearly to zero at end of the fiber [15]. The mean curvature is $3 \mathrm{~s} /\left(2 \mathrm{~L}^{2}\right)$, where $s$ is the distance the exit end was deflected, and $L$ the length of the bent portion of the fiber. To prevent the beam passing directly from the source to the detector, a lead pinhole collimator with a diameter of $350 \mu \mathrm{m}$, which is smaller than the fiber diameter, was aligned with the entrance end of the polycapillary fiber. The polycapillary holding apparatus was mounted on rotational stages, that could rotate about both axes perpendicular to the fiber axis. Each fiber was aligned with the $x$-ray beam by rotating the fiber about both axes until the transmission was maximized.

The transmission efficiency, defined as the ratio of the photon flux at the exit end of the fiber to the photon flux at the entrance of the fiber, is limited by the fractional open area 
of the polycapillary fiber. Transmission efficiencies of $57.3 \%$ and $54.5 \%$ for $22 \mathrm{keV}$ and 44 $\mathrm{keV}$ photons, respectively, were obtained for a 120 -mm-long straight fiber aligned with the incident bear. These values are close to the fractional open area of the fiber $(\sim 60 \%)$.

We measured the transmission efficiency as a function of the tilt angle between the incident beam and the axis of the fiber. Figure 2 compares the measurements with the raytracing simulation. Transmission efficiency decreases with the tilt angle because the average angle between the photon trajectories and the reflecting surface increases, producing greater absorption losses. The larger mean angle also causes more reflections to take place as the photons travel the length of the polycapillary fiber. The results show that the full width at half maximum of the transmission curve for $22 \mathrm{keV}$ photons is about twice that for $44 \mathrm{keV}$ photons because the critical angle for $22 \mathrm{keV}$ photons is twice that for $44 \mathrm{keV}$ photons. The measured transmission curves are only slightly lower than the simulated ones, which shows that the reflectivity in the polycapillary fiber is close to the theoretical values used in the simulations.

We also measured the transmission efficiency through a 114-mm-long bent polycapillary fiber. As described above, the fiber was bent by aligning the straight entrance portion with the incident beam and deflecting the exit end with a micrometer to different positions. Figure 3 compares the transmission efficiency as a function of mean curvature for $22 \mathrm{keV}$ and $44 \mathrm{keV}$ photons with the simulation. Some of the $\mathrm{x}$-ray trajectories transmitted through the straight portion of the fiber enter the bent portion with incident grazing angles exceeding the critical angle. As the degree of bending increases, the fraction of these trajectories also increases, which results in a decrease in the transmission (Figure 3). The results show that lower energy photons can be deflected more easily because of their larger critical angle.

The results show that glass capillaries can effectively guide and bend $x$ rays up to 44 $\mathrm{keV}$. The agreement between the measured data and simulations indicates that the reflectivity in the polycapillary fiber is close to the theoretical values used in the simulations. The large ratio of the transmission efficiencies between the $22 \mathrm{keV}$ and $44 \mathrm{keV} \times$ rays when the fiber is bent or tilted away from the incident beam shows that the technique of filtrating high-energy $x$ rays using capillaries is quite promising, especially with divergent beams for which capillaries can capture a larger solid angle of lower energy photons, while effectively filtrating 
undesired higher energy spectra.

We are grateful to Muradin A. Kumakhov, Walter M. Gibson, Mark L. Rivers, and L. Dean Chapman for their support, and to Avril D. Woodhead for her comments on the manuscript. This research was partially supported by the U. S. Department of Energy under contract Numbers DE-FG02-91ER81221 and DE-AC02-76CH0016. 


\section{References}

1. P. B. Hirsch and J. Kellar, Proc. Phys. Soc. (London) B64, 369, (1951).

2. D. Mosher and S. Stephanakins, Appl. Phys. Lett., 29, 105, (1976).

3. Pound and Rebka, Phys. Rev. Lett., 3, 439, (1959).

4. J. Marton, Appl. Phys. Lett., 9, 194, (1966).

5. P. S. Chung and R. Pantell, Electron Lett., 13, 527,(1977).

6. E. A. Stern, Z. Kalman, A. Lewis, and K. Lieberman, Appl. Opt. 27, 5135$5139,(1988)$.

7. D. A. Carpenter and M. A. Taylor, Adv. in X-Ray Anal. Vol. 34, 217 (1991).

8. M. A. Kumakhov, Cert. of Authorship 1322888, USSR, 1984.

9. V. A. Arkadiev, A. I. Kolomitsev, M. A. Kumakhov, V. V. Labuzov, Yu. P. Chertov, and I. M. Shakhparonov, Poverkhnost, 2, 44, (1987).

10. M. A. Kumakhov and F. F. Komanov, Phys. Rep. 191, 290-350, 1990.

11. V. E. Kovantsev, J. Pant, V. Pantojas, N. Nazaryan, T. M. Hayes, and P. D. Persans, Appl. Phys. Lett., 62, 2905-2907, (1993).

12. M. Vartanian, D. Gibson, R. D. Frankel, and J. Drumheller, (in press), Proc. SPIE Conference on Microlithography, San Jose, March 1993.

13. Q. F. Xiao, I. Yu. Ponomarev, A. I. Kolomitsev, and J. C. Kimbal, SPIE Proc., 1736, 227-238, (ed. R. B. Hoover), (1992).

14. J. B. Ullrich, V. Kovantzev, and C. A. MacDonald, to be (in press), J. Appl. Phys. (1993).

15. D. J. Barber and R. Loudon, "An Introduction to the Properties of Condensed Matter", Cambridge University Press (1989). 


\section{Figure Captions}

Figure 1. Optical micrograph of the cross-section of a polycapillary fiber used in this study.

Figure 2. Measured and simulated transmission efficiency as a function of the angle between the capillary axis and the incident beam with energies of $22 \mathrm{keV}$ and $44 \mathrm{keV}$ for a 120-mm-long fiber.

Figure 3. Measured and simulated transmission efficiency as a function of mean curvature for $22 \mathrm{keV}$ and $44 \mathrm{keV} x$ rays for a $114-\mathrm{mm}-$ long fiber. 


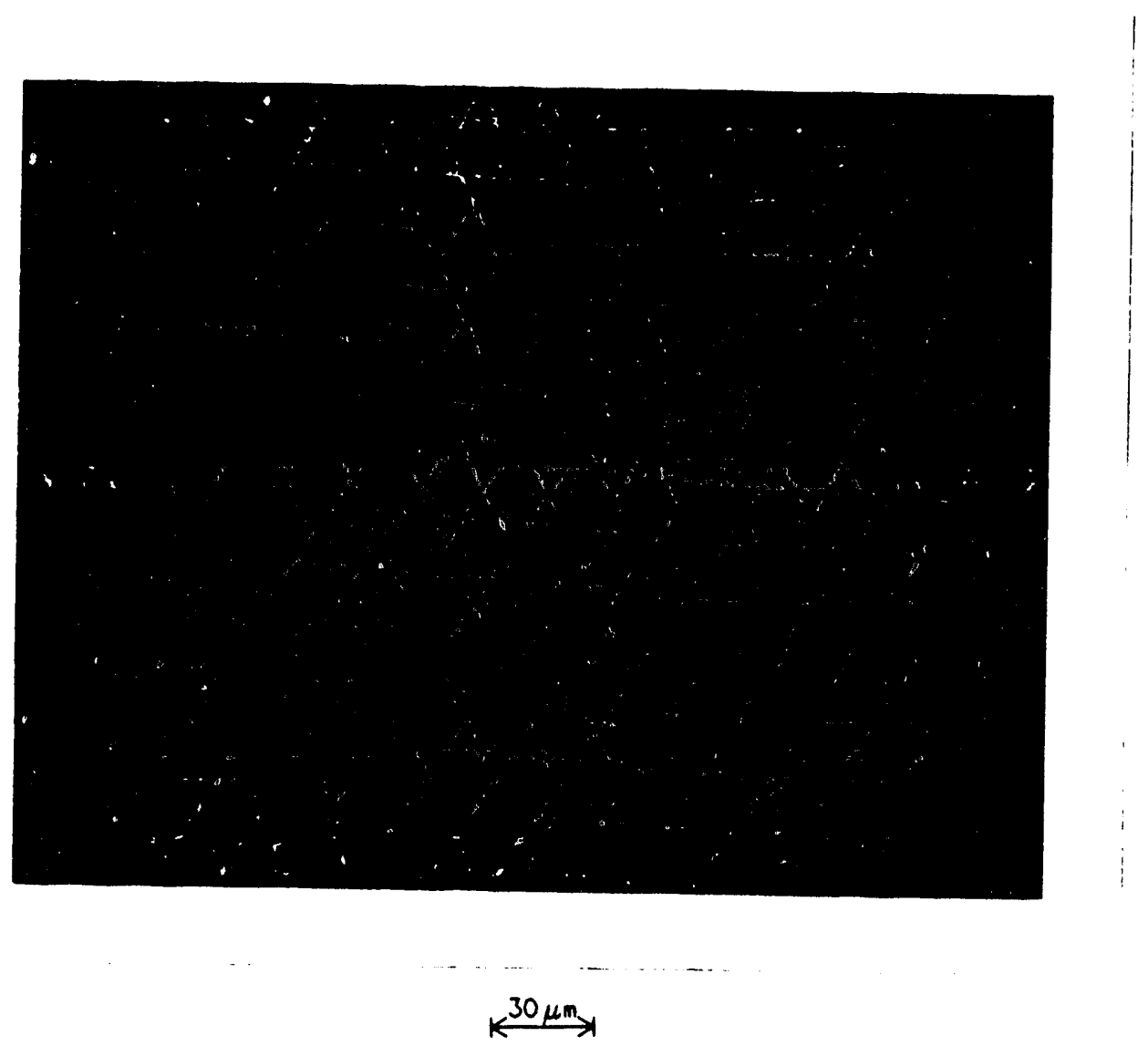




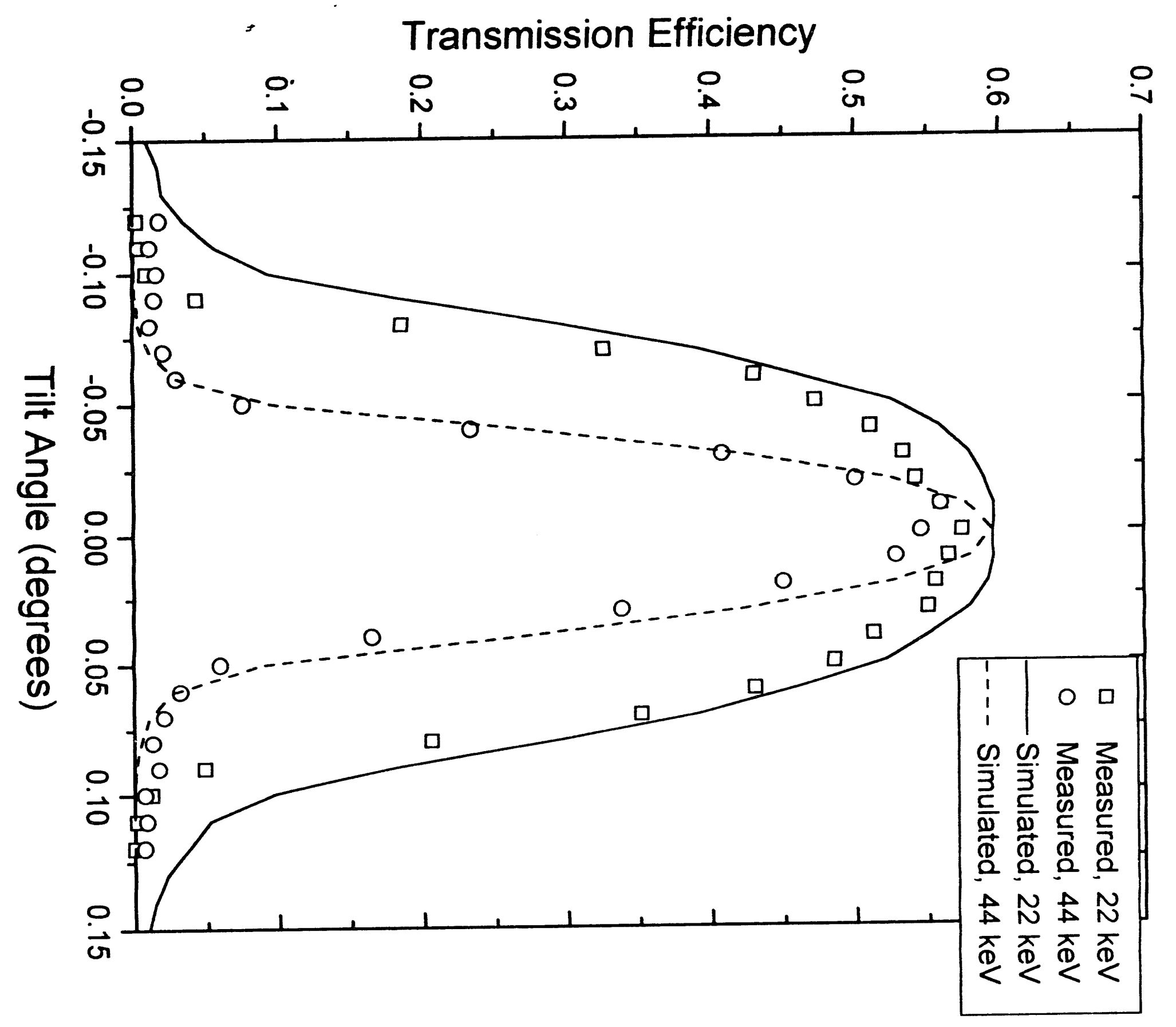




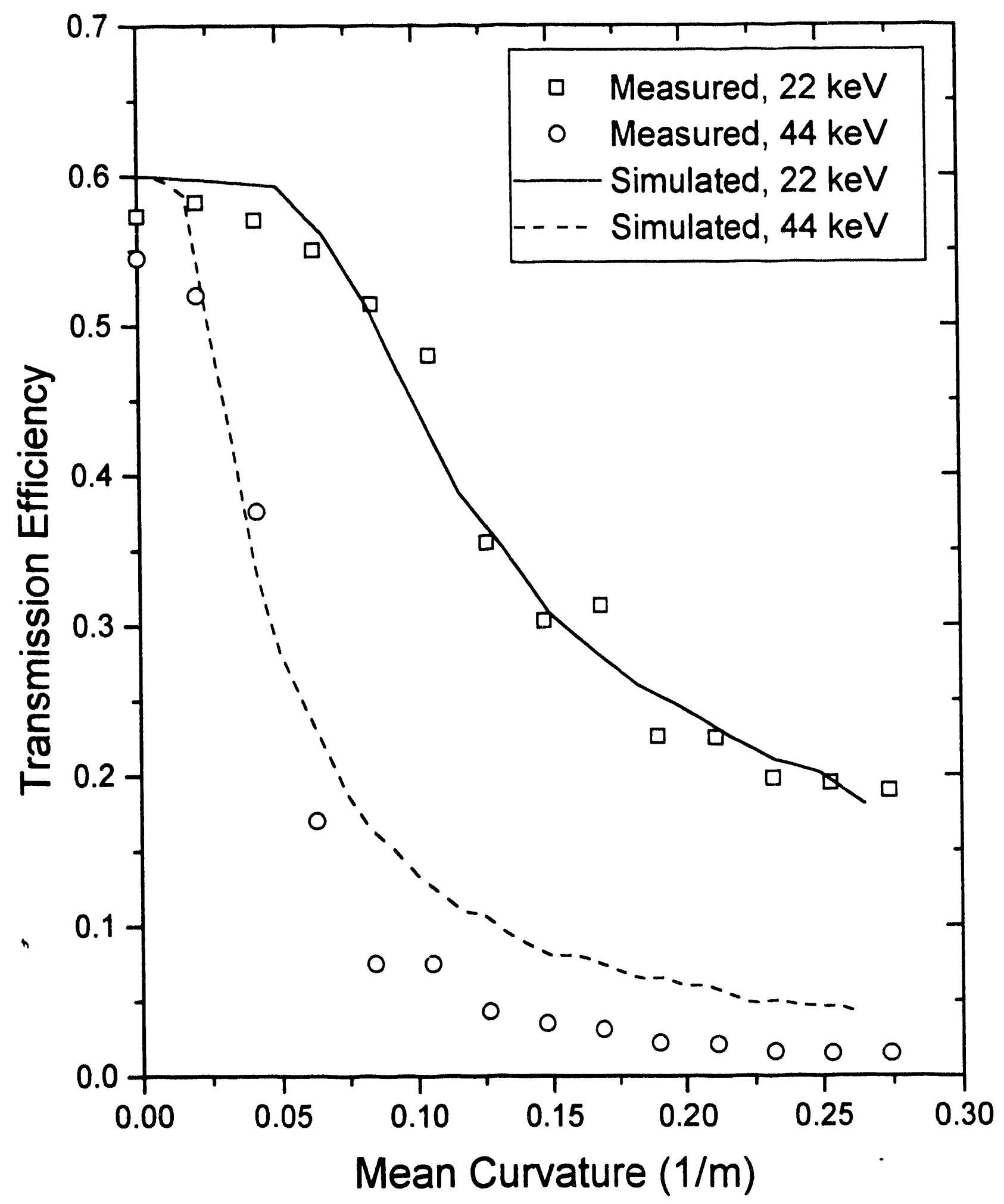


11
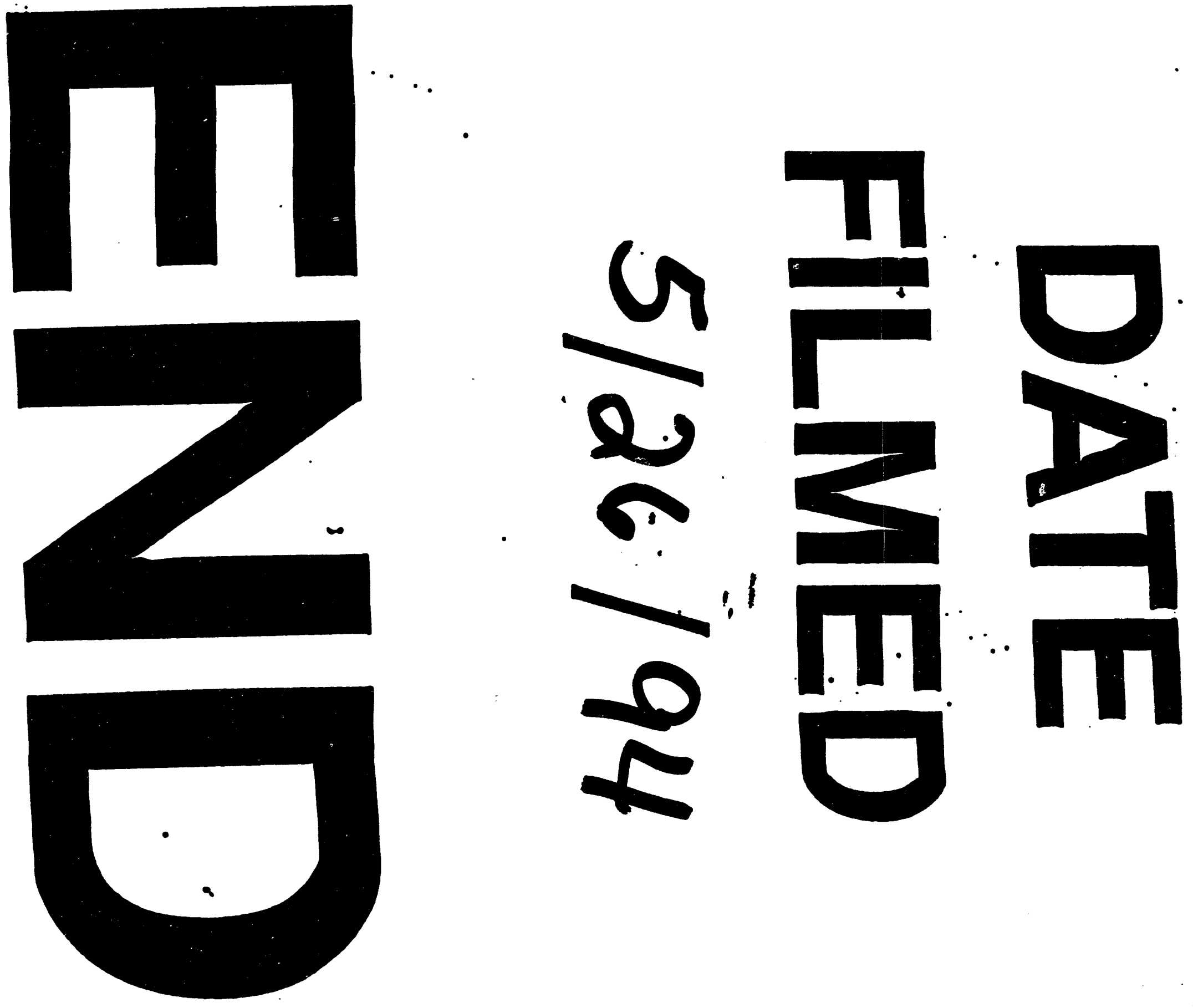
